\title{
Novel derivative of aminobenzenesulfonamide (3c) induces apoptosis in colorectal cancer cells through ROS generation and inhibits cell migration
}

Khayal Al-Khayal ${ }^{1}$, Ahmed Alafeefy ${ }^{2}$, Mansoor-Ali Vaali-Mohammed ${ }^{1}$, Amer Mahmood ${ }^{3}$, Ahmed Zubaidi ${ }^{1}$, Omar Al-Obeed ${ }^{1}$, Zahid Khan ${ }^{4}$, Maha Abdulla ${ }^{1}$ and Rehan Ahmad ${ }^{1 *}$

\begin{abstract}
Background: Colorectal cancer (CRC) is the $3^{\text {rd }}$ most common type of cancer worldwide. New anti-cancer agents are needed for treating late stage colorectal cancer as most of the deaths occur due to cancer metastasis. A recently developed compound, $3 \mathrm{c}$ has shown to have potent antitumor effect; however the mechanism underlying the antitumor effect remains unknown.

Methods: 3c-induced inhibition of proliferation was measured in the absence and presence NAC using MTT in HT-29 and SW620 cells and XCELLigence RTCA DP instrument. 3c-induced apoptotic studies were performed using flow cytometry. 3c-induced redox alterations were measured by ROS production using fluorescence plate reader and flow cytometry and mitochondrial membrane potential by flow cytometry; NADPH and GSH levels were determined by colorimetric assays. Bcl2 family protein expression and cytochrome $\mathrm{c}$ release and PARP activation was done by western blotting. Caspase activation was measured by ELISA. Cell migration assay was done using the real time xCELLigence RTCA DP system in SW620 cells and wound healing assay in HT-29.

Results: Many anticancer therapeutics exert their effects by inducing reactive oxygen species (ROS). In this study, we demonstrate that $3 \mathrm{c}$-induced inhibition of cell proliferation is reversed by the antioxidant, $\mathrm{N}$-acetylcysteine, suggesting that $3 \mathrm{C}$ acts via increased production of ROS in HT-29 cells. This was confirmed by the direct measurement of ROS in 3c-treated colorectal cancer cells. Additionally, treatment with $3 \mathrm{c}$ resulted in decreased NADPH and glutathione levels in HT-29 cells. Further, investigation of the apoptotic pathway showed increased release of cytochrome $\mathrm{c}$ resulting in the activation of caspase-9, which in turn activated caspase- 3 and $-6.3 \mathrm{c}$ also (i) increased p53 and Bax expression, (ii) decreased Bcl2 and BclxL expression and (iii) induced PARP cleavage in human colorectal cancer cells. Confirming our observations, NAC significantly inhibited induction of apoptosis, ROS production, cytochrome $\mathrm{c}$ release and PARP cleavage. The results further demonstrate that $3 \mathrm{c}$ inhibits cell migration by modulating EMT markers and inhibiting TGFß-induced phosphorylation of Smad2 and Samd3.
\end{abstract}

Conclusions: Our findings thus demonstrate that $3 c$ disrupts redox balance in colorectal cancer cells and support the notion that this agent may be effective for the treatment of colorectal cancer.

Keywords: Colorectal cancer, ROS, NAC, Apoptosis, Cell migration

\footnotetext{
* Correspondence: arehan@ksu.edu.sa

${ }^{1}$ Colorectal Research Center, Department of Surgery, King Khalid University

Hospital College of Medicine, King Saud University, PO BOX 7805 (37),

Riyadh, Saudi Arabia

Full list of author information is available at the end of the article
} 


\section{Background}

Colorectal cancer (CRC) is the second leading cause of cancer-related deaths in the US and is associated with high mortality. CRC is the $3^{\text {rd }}$ most common cause of cancer globally [1]. The basis for the high mortality in patients with colorectal cancer is the formation of distant metastasis. Colorectal cancer patients diagnosed at early stage have a 5 year-survival rate of about $90 \%$, which decreases to $65 \%$ with lymph node metastasis and to $<10 \%$ with distant metastasis [2]. CRC is a heterogenous disease with progressive accumulation of genetic and epigenetic alterations [3]. Oncogene activation and loss of tumor suppressor genes are crucial for transformation from normal cells to cancer cells [4].

Oxidative stress has been shown to be involved in diverse physiological and certain pathological conditions, such as cancer [5]. Reactive oxygen species (ROS) are produced in a cell as a result of normal metabolic processes, as well as xenobiotic exposures. The concentration of ROS determines its beneficial or harmful effects for the cells and tissues [6]. Owing to their basal higher ROS levels as compared to normal cells, cancer cells are more susceptible when encountering additional ROS insults induced by anticancer agents [7]. Excess levels of ROS can trigger cell death by activating pathways leading to apoptosis, necrosis and autophagy [8,9]. Various studies have reported activation of p38 MAPK and JNK pathways in ROS mediated apoptotic cell death [10-12]. Moreover, Akt has been shown to be regulated by ROS $[13,14]$.

Elevated levels of ROS promote genotoxic damage and thereby cancer progression by amplifying genomic instability and also by stimulating tumor promoting signaling pathways [15]. Consequently, oxidative stress provides a growth advantage to transformed cells by activating signaling pathways that stimulate proliferation and maintenance of cancer cells. However, excessive levels of ROS can have deleterious effect on cancer cells and can readily induce cell cycle arrest and apoptosis. As a result, selectively targeting cancer cells by modulating ROS levels has been proposed as an effective therapeutic strategy. Several studies showed effect of anticancer agents that increases the ROS levels to efficiently kill cancer cells $[16,17]$. Abnormal increases in the reactive oxygen species during the oncogenic transformation process render cancer cells sensitive to oxidative stress inducing agents [18]. These agents by further elevating ROS levels beyond the antioxidant capacity of the malignant cells induce apoptosis. However, these ROS inducers have no or minimal effects on normal cells due to their low production of ROS and high antioxidant capacity. Thus, oxidative stress in cancer cells has the potential to be exploited in the development of novel and selective anticancer therapeutics. Anti-cancer drugs being used for the treatment of colorectal cancer include 5-flurouracil, oxaliplatin and irinotecan [4]. The treatment involve conventional therapy which include surgical resection, chemotherapy and radiation, all of them are often inadequate in treating colorectal cancer. Therefore, new treatment options are urgently needed. Despite the discovery of novel targeted agents and use of different combination therapeutics, no treatment regimens are available for treating colorectal cancer patients with distant metastasis.

Recently, we discovered a novel derivative of aminobenzenesulfonamide (2-substituted-quinazolin-4-yl-aminobenzenesulfonamide), designated $3 c$, as a potential anti-tumor agent [19]. The aim of the present study was to investigate the mechanism of 3c-induced inhibition of cellular proliferation. This study demonstrated that inhibitor $3 \mathrm{c}$ induces apoptosis mediated by increased production of reactive oxygen species in colorectal cancer cells. Furthermore, $3 \mathrm{c}$ reduces NADPH and GSH levels along with up-regulation of cytochrome c, cleaved PARP and activation of caspases. In addition, the small molecule $3 \mathrm{c}$ inhibited the cell migration of colorectal cancer cells. These findings identify ROS induction as the primary mechanism of action of compound 3c and support the development and use of oxidative stress inducers as anticancer agents.

\section{Methods}

\section{Cell culture}

Human HT-29 and SW620 colorectal cancer cells were obtained from ATCC (Manassas VA) and grown in RPMI (Invitrogen) containing 10\% heat-inactivated fetal bovine serum, $100 \mu \mathrm{g} / \mathrm{ml}$ streptomycin, 100 units/ ml penicillin and $2 \mathrm{mmol} / \mathrm{l} \mathrm{L}$-glutamine. In certain experiment TGF $\beta$ stimulation was done at $10 \mathrm{ng} / \mathrm{ml}$.

\section{Cell viability assay}

Cell viability was determined using MTT [19]. Briefly, after culturing cells in 96 well plate for $24 \mathrm{~h}$, they were treated with $3 \mathrm{c}(5 \mu \mathrm{M})$ for $24 \mathrm{~h}$. Freshly prepared $10 \mu \mathrm{L}$ of MTT 3(4, 5-dimethylthiazolyl-2)-2, 5-diphenyltetrazolium bromide) $(5 \mathrm{mM})$ solutions were added to the cells and was further incubated for $2 \mathrm{~h}$ at $37{ }^{\circ} \mathrm{C}$ in $5 \% \mathrm{CO} 2.100 \mu \mathrm{L}$ of dimethyl sulfoxide (DMSO) were added in each well to dissolve the crystal of formazan, which formed in the reaction of MTT at the time of incubation. The crystals were dissolved through pipetting carefully. The absorbance of the product was measured at $540 \mathrm{~nm}$ using a microplate reader. The experiments were performed in triplicates for each condition. The graph illustrates the mean and standard deviation (SD) values of three independent experiments.

\section{Cytotoxicity assay using xCELLigence system}

Optimal seeding concentration for proliferation of HT-29 was determined. HT-29 cells (5000 cell in $150 \mu \mathrm{L}$ medium/ well) were seeded in 16 well plates (E-plate 16 ACEA Biosciences Inc, San Diego USA) following the xCELLigence Real Time Cell Analyzer (RTCA) DP instrument manual as 
provided by the manufacturer. After $24 \mathrm{~h} 3 \mathrm{c}(5 \mu \mathrm{M})$ was added and the experiment was allowed to run for 3-4 days. Baseline cell index were calculated for at least two measurements from three replicate experiments. Appropriate wells were pre-treated with NAC $(5 \mathrm{mM})$ for $1 \mathrm{~h}$ and then compound $3 \mathrm{c}(5 \mu \mathrm{M})$ was added. Cell proliferation was monitored for another $72 \mathrm{~h}$.

\section{Apoptosis}

Cells treated with compound $3 \mathrm{c}$ in the presence and absence of NAC was incubated with propidium iodide (PI)/annexin V-FITC (BD BioSciences) for $15 \mathrm{~min}$ at room temperature and then analyzed by flow cytometry on FACSCalibur (BD Biosciences).

\section{Western blotting}

Whole cell lysates were prepares using RIPA lysis buffer as described [20]. Total protein concentration was determined using Bradford Protein reagent (Bio-Rad). Soluble proteins were loaded on precast TGX gels and were analyzed by immunoblotting with anti-cytochrome c (1:200 Abcam), anti-PARP (1:200 BioVision), anti-Bax, anti-Bcl2, antiBclxL, anti-Cyclin D1 (Dilution 1:1000; Santa Cruz Biotechnology) and anti- $\beta$-actin (1:10,000 Sigma). Reactivity was detected with horseradish peroxidase-conjugated secondary antibodies and chemiluminescence by Clarity Western ECL Substrate (Bio-Rad). Membrane was developed using CDigit Blot Scanner (LI-COR, Hamburg Germany).

\section{Cytochrome C measurement}

Briefly, cells were treated with $3 \mathrm{c}$ for $18 \mathrm{~h}$ and harvested cells were homogenized in $1 \mathrm{X}$ cytosolic extraction buffer by 30 strokes of a Dounce homogenizer using mitochondria isolation Kit (Abcam ab65311). The homogenate was centrifuged at $600 \times g$ for $5 \mathrm{~min}$, and the resulting supernatant was centrifuged at $10,000 \times g$ for $10 \mathrm{~min}$. The mitochondrial pellet was washed with the buffer and resuspended in mitochondrial extraction buffer. Mitochondria and cytosolic extracts were immunoblotted for cytochrome c.

\section{Reactive Oxygen Species (ROS) measurement}

Intracellular ROS accumulation was monitored in HT-29 cells by adding the $\mathrm{H}_{2}$-DCFDA [21]. In brief, 5000 cells/ well were seeded with phenol free DMEM in a 96-well microplate. The cells were treated with $3 \mathrm{c}$ for $18 \mathrm{~h}$. DCFDA was added to the wells at $5 \mu \mathrm{M}$ for $30 \mathrm{~min}$. Increases in fluorescence were measured at excitation and emission wavelengths of 485 and $535 \mathrm{~nm}$, respectively.

\section{ROS measurement by flow cytometry}

Cells were pretreated with compound $3 c(5 \mu \mathrm{M})$ for different time points. Cells were then treated with $\mathrm{c}-\mathrm{H}_{2}$ DCFDA $(5 \mathrm{uM})$ for $20 \mathrm{~min}$ at $37 \mathrm{C}$ to assess hydrogen peroxide (H2O2)-mediated oxidation to fluorescent compound DCF
[22]. Fluorescence of oxidized DCF was measured using flow cytometry (BD FACS Calibur) at excitation wavelength of $480 \mathrm{~nm}$ and emission wavelength of $525 \mathrm{~nm}$.

\section{Measurement of mitochondrial membrane potential}

Cells were treated with $3 \mathrm{c}(5 \mathrm{uM})$ for different time points then cells were incubated with rhodamine $123(25 \mathrm{ng} / \mathrm{ml})$ (Molecular Probes) in PBS for $20 \mathrm{~min}$ at 37C. Rhodamine 123 positive populations were monitored using flow cytometry [22].

\section{GSH measurement}

The levels of GSH in the cells were determined according to the method based on the formation of 2-nitro-5-tiobenzoic acid from DTNB in the presence of GSH [21]. In brief, $25 \mu \mathrm{l}$ of trichloroacetic acid (15\%) was added to $50 \mu \mathrm{l}$ of the homogenate, followed by centrifugation at $13,000 \times g$ for $5 \mathrm{~min}$ at $4{ }^{\circ} \mathrm{C}$. A supernatant aliquot $(50 \mu \mathrm{l})$ was mixed with $50 \mu \mathrm{l}$ of $3.4 \mathrm{mM}$ ethylenediaminetetraacetic acid (EDTA) dissolved in PBS, $1 \mathrm{ml}$ of PBS, and $250 \mu \mathrm{l}$ of DTNB in PBS $(20 \mathrm{mg} / \mathrm{ml})$. The absorbance was measured at $412 \mathrm{~nm}$ after $15 \mathrm{~min}$ and compared to a standard curve of GSH (0.01-0.5 mM).

\section{Determination of NADPH levels}

Intracellular NADPH concentrations were measured using the NADP/NADPH Assay Kit as per the manufacturer's instructions (BioVision, Milpitas, CA USA).

\section{Caspase activity assay}

Caspase activity assay was determined using Caspase Colorimetric Protease Assay Sample Kit for measuring Caspase-2, -3, -6, -8, -9 (Invitrogen KHZ1001) at $400 \mathrm{~nm}$ on microplate reader.

\section{Cell migration assay}

For monitoring of cell migration in real-time the xCELLigence Real Time Cell Analyzer Dual Plate (RTCA-DP) instrument was used according to the manufacturer's recommendations (Acea Biosciences Inc USA). The impedance is expressed as a dimensionless parameter, termed cell index, and is directly proportional to the area covered by cells. For detection of cellular migration, electrical impedance changes are measured at a gold microelectrode plated on the bottom of a membrane separating the upper and lower chambers. The SW620 cell line was treated without and with $3 \mathrm{c}$ and subjected to serum starvation $12 \mathrm{~h}$ before the start of measurement. For cell migration assays, $12 \mathrm{x}$ $10^{3}$ cells in RPMI-1640 were seeded per well of a 16-well CIM plate, and the lower chamber was loaded with RPMI1640 supplemented with 10\% FCS. Cell index values were monitored every $5 \mathrm{~min}$ for $150 \mathrm{~h}$. At least three independent experiments were performed for monitoring cell migration each carried out in triplicates. Cell index values were 
calculated and plotted using the RTCA software 1.2.1 of the RTCA xCELLigence system. For cell migration, the cell index curves were monitored for $150 \mathrm{~h}$.

\section{Wound healing assay}

Wound healing assay was performed as described by Li et al. [23]. HT-29 cells were seeded in at $1 \times 10^{6}$ cells/well in duplication. Cells were allowed to grow to near confluency for three days and scratched a wound through the center of the well. Washed with PBS three times, DMSO and 3c compound was added in duplicate. After taking pictures under microscope (10X), plates were transferred to $\mathrm{CO} 2$ incubator at $37{ }^{\circ} \mathrm{C}$ and incubated for $48 \mathrm{~h}$. After final incubation, pictures were again taken under microscope.

\section{Results}

$3 c$ induced inhibition of cell proliferation is reversed by NAC Compound $3 \mathrm{c}$ is a novel derivative of aminobenezenesulfonamide that has been shown to inhibit cellular proliferation in colorectal cancer cells [19]. ROS is a major signaling molecule mediating the effect of anticancer therapeutics. Hence, in this study, we investigated whether 3c induced inhibition of cell proliferation is mediated by reactive oxygen species (ROS). Compound 3c had significant cytotoxic effects on HT-29 colorectal cancer cells that showed a marked reduction in viability to $18 \%$ following $24 \mathrm{~h}$ treatment with the drug. Noticeably, the antioxidant $\mathrm{N}$-acetylcysteine (NAC) was found to significantly reverse the effect of 3c (Fig. 1a). Similar result was obtained in another colorectal cancer cells, SW620 (Additional file 1: Figure S1). This finding was confirmed by using the xCELLigence real time cell proliferation system, whereby NAC as observed earlier was found to inhibit the cytotoxicity induced by $3 \mathrm{c}$ in colon cancer cells (Fig. 1b). To further investigate whether 3c mediated cell death is caused by apoptosis or necrosis, we performed flow cytometry on HT-29 colorectal cancer cells. As shown in Fig. 1c, 3c treatment significantly induced early apoptosis in a dose dependent manner (Fig. 1c). Pretreatment of the cancer cells with NAC markedly blocked the induction of apoptosis supporting the role of oxidative stress (Fig. 1d). These findings thus indicate that $3 \mathrm{c}$ induced inhibition of cell proliferation is mediated by ROS. To compare the effect of 3c with standard drug, doxorubicin is known to inhibit HT-29 cell proliferation. Herein doxorubicin was found to decrease cell viability in dose dependent manner. 3c significantly potentiated Dox-mediated inhibition of cell viability (Additional file 1: Figure S2). Of note 3c has no cytotoxic effect on human mammary epithelial cells MCF10A (Additional file 1: Figure S3). These findings confirmed that $3 c$ exerts cytotoxic effect in cancer cells having no or minimal effect on normal cells.

\section{3c modulates redox balance}

To assess whether compound $3 \mathrm{c}$ induces oxidative stress, we utilized the property of $2^{\prime}, 7^{\prime}$-dichlorofluorescin diacetate (DCFDA) fluorogenic dye which after oxidization emits green fluorescence. Treatment of HT-29 cells with $3 \mathrm{c}$ inhibitor was associated with increases in ROS as compared to the untreated control (Fig. 2a Left). Production of ROS by 3c was again confirmed using flow cytometry (Fig. 2a Right). 3c was found to enhance ROS production in metastatic colorectal cancer cell line, SW620 as well (Additional file 1: Figure S4). Treatment of cells with 3c was associated with increases in hydrogen peroxide in a time dependent manner that reached maximal levels at $12 \mathrm{~h}$ (Fig. 2b). The induction of ROS by $3 \mathrm{c}$ was reversed by NAC (Fig. 2c). A major source of ROS is produced by the mitochondria electron transport chain. Moreover, oxidative stress inhibits the mitochondrial membrane potential. In this regard, rhodamine 123 staining of HT-29 cells demonstrated that $3 \mathrm{c}$ decreases the mitochondrial membrane potential in a time dependent manner (Fig. 2d). Similarly $3 \mathrm{c}$ also inhibited mitochondrial membrane potential in SW620 cells (Additional file 1: Figure S5). Treatment with $3 \mathrm{c}$ was also associated with reduction in GSH and NADPH levels (Fig. 2e). In summary, 3c results in increased levels of ROS and decreased NADPH and GSH. These findings thus indicate that $3 \mathrm{c}$ disrupts redox balance in colorectal cancer cells with increases in hydrogen peroxide and decreases in NADPH and GSH.

\section{3c-induces modulation of $\mathrm{BCl} 2$ family protein and inhibits Cyclin D1}

The interplay between the pro-apoptotic Bax and antiapoptotic $\mathrm{Bcl} 2$ proteins results in the release of cytochrome c from mitochondria, which leads to caspase activation and subsequent apoptosis. Incubation of cells with $3 \mathrm{c}$ increased expression of Bax (Fig. 3a). Additionally, 3c treatment inhibited the expression of the anti-apoptotic $\mathrm{Bcl} 2$ and $\mathrm{BclxL}$ proteins. Similar results were obtained from studies with the metastatic colorectal cancer cell line SW620 (Fig. 3b). Cyclin D1 is a major proliferation gene known to regulate cell cycle progression. 3c resulted in the inhibition of cyclin D1 expression in dose dependent manner (Fig. 3c). To understand the mechanism for Bax induction, p53 is a well-known tumor suppressor involved in apoptosis, cell cycle regulation and DNA repair. Bax and $\mathrm{Bcl} 2$ are transcriptional targets of p53 [24]. p53 has been shown to induce Bax oligomerization and cytochrome c release from mitochondria. Notably, treatment of cells with 3c increased p53 expression in a time dependent manner (Fig. 3d). Bax expression was also found to be increased and $\mathrm{Bcl} 2$ expression was decreased. These findings indicate that $3 \mathrm{c}$ modulates $\mathrm{Bcl}$ family proteins by increasing Bax expression and inhibiting antiapoptotic proteins favoring the balance towards apoptosis along with inhibiting cell cycle regulator cyclin D1. 


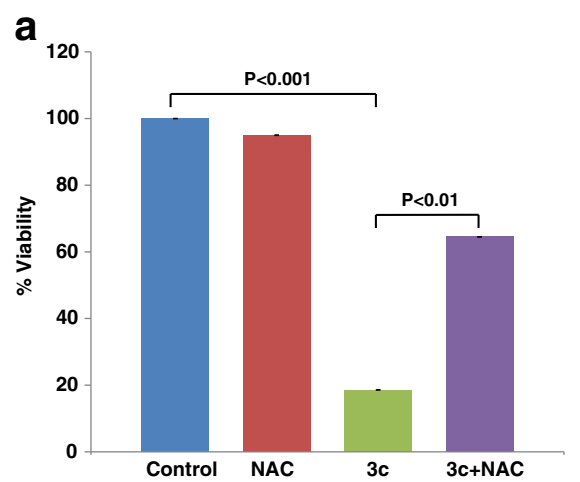

b

C
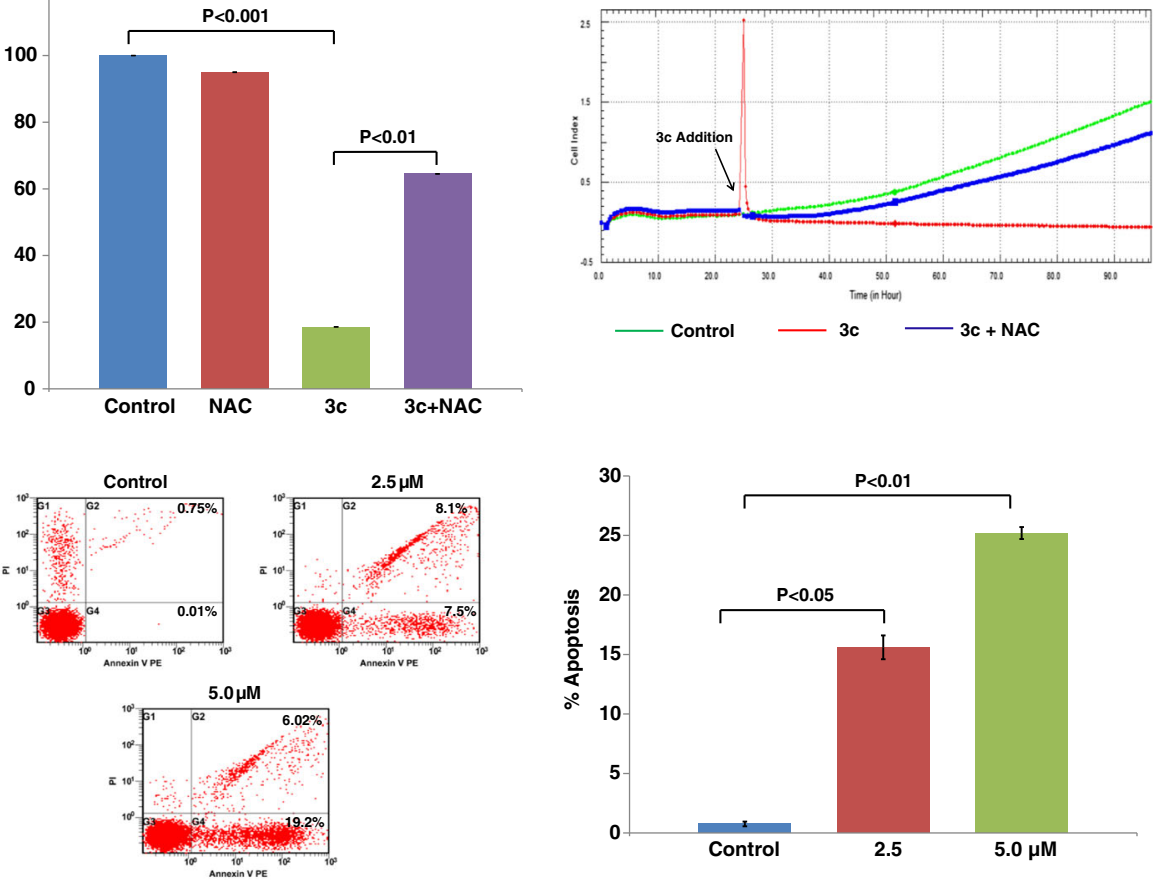

Control $-3 \mathrm{c}-3 \mathrm{C}+\mathrm{NAC}$

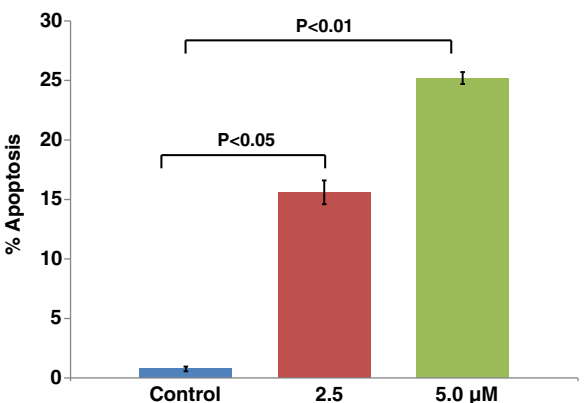

d
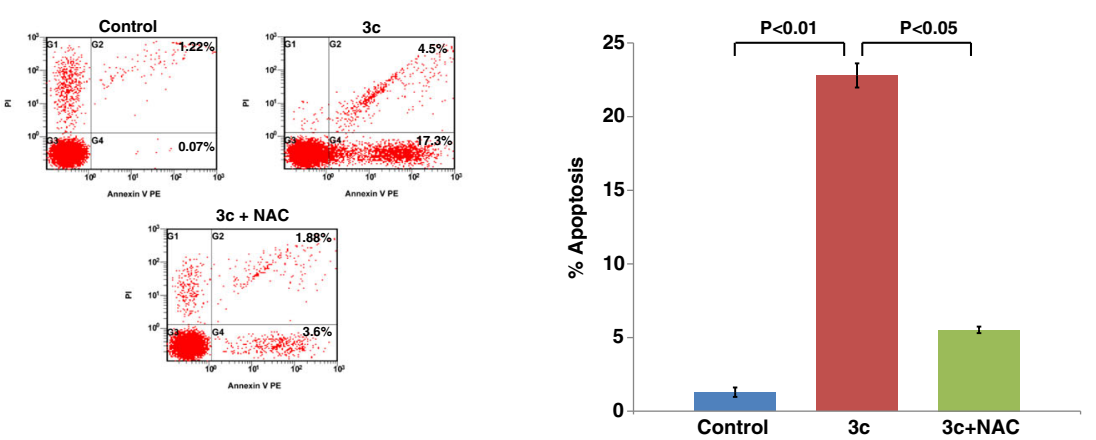

Fig. 1 NAC reverses the cytotoxicity induced by $3 c$. a. HT-29 cells were treated with $3 c(5 \mu M)$ in the absence and presence of NAC (5 mM), Cell viability was measured by MTT assay. The results are expressed as mean of 3 independent determinations (mean \pm SD). b. $5 \times 10^{3}$ cells were seeded in E-plate 16, after overnight incubation, cells were pre-treated with NAC followed by treatment with 3c. Real-time proliferation monitoring of HT-29 cells was performed by measuring cell index to evaluate the cytotoxic effect of $3 c$ using xCELLigence RTCA-DP system. c-d. HT-29 cells were treated with various concentration of $3 \mathrm{c}$ for 24 h. $0.5 \%$ DMSO was used as a control. Cells were processed for flow cytometry using Annexin V/PI staining. The percentage of Annexin V+ population indicates apoptosis induction. Results shown are representative of 3 independent experiments. In some cases where indicated cells were pre-treated with NAC for $1 \mathrm{~h}$ followed by $3 \mathrm{c}$ treatment

\section{3c activates Cytochrome c release, PARP cleavage and \\ Caspase cascade}

There are two major classes of initiator (i.e., caspase-9) and effector caspases (i.e., Caspases-3 and -6). Cytochrome c release from mitochondria binds with APAF1 and ATP leading to the activation of the initiator caspase-9, which in turn activates the effector caspase- 3 and 6 . Involvement of cytochrome c release from mitochondria is an indicator of activation of the intrinsic apoptotic pathway. Treatment with 3c induces cytochrome $\mathrm{c}$ in human colorectal cancer cells (Fig. 4a). Similar results were also obtained in SW620 colorectal cancer cells (Fig. 4b). Furthermore, 3c induces cytochrome c release from mitochondria into the cytosol (Fig. 4c). To investigate which caspase pathway is activated by $3 \mathrm{c}$ for induction of apoptosis, ELISA was performed with various caspase substrates. HT-29 cells treated with 3c exhibited enhanced activation of caspase-9; thereby activating effector caspase-3 and 6 (Fig. 4d). A modest increase in caspase- 8 activity was also observed. This result indicates that $3 \mathrm{c}$ induced apoptosis is mediated predominantly by the intrinsic apoptosis pathway and to a limited extent the extrinsic pathway. PARP (Poly-ADP-ribose polymerase) is a family of proteins known to be involved in various cellular processes like DNA repair and programmed cell death. 

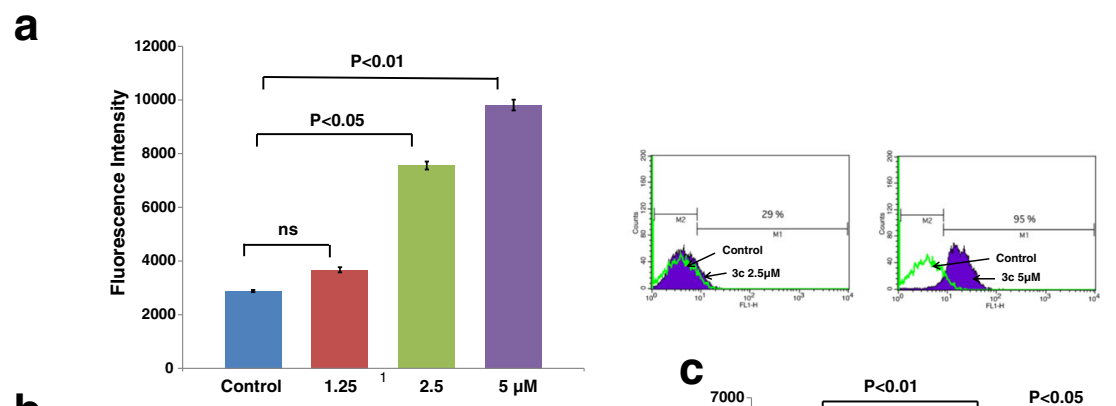

\section{b}
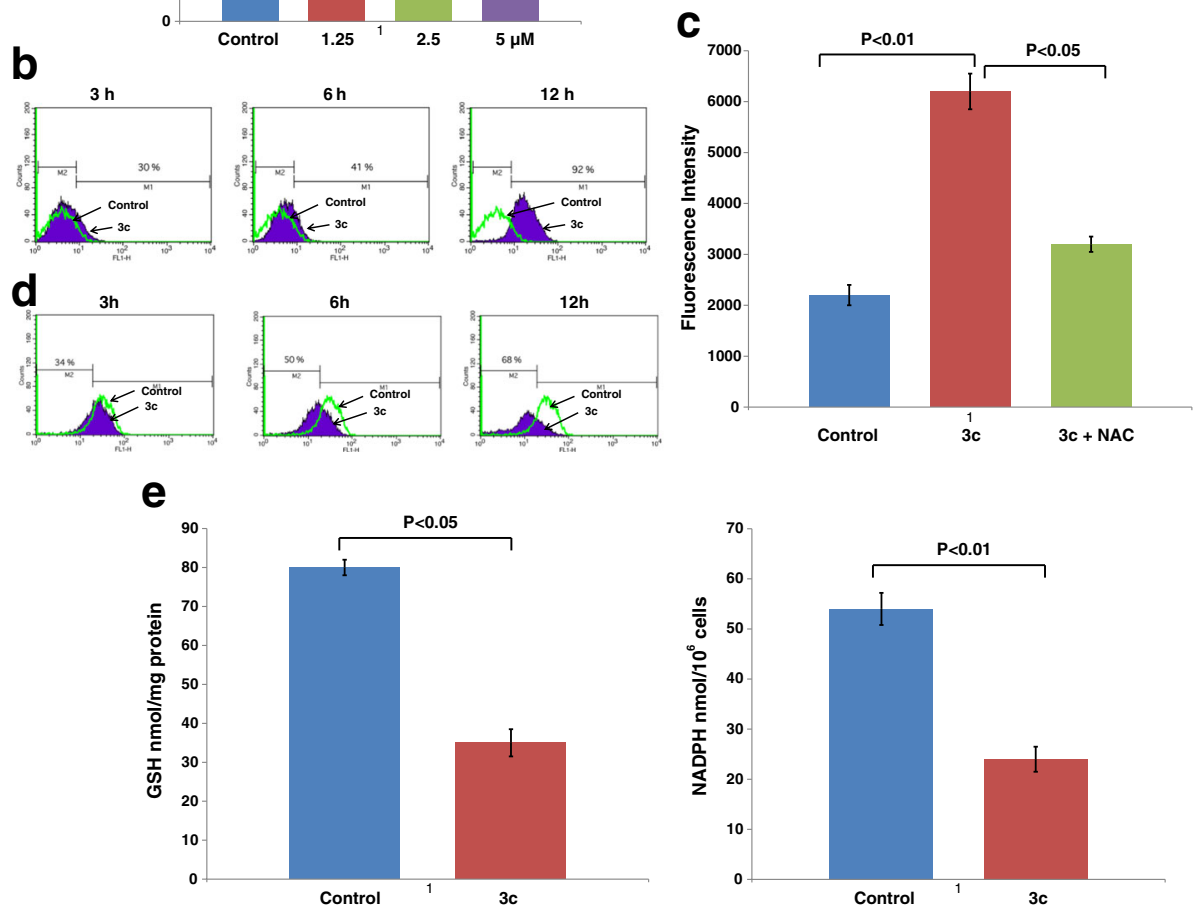

Fig. 2 Compound $3 c$ modulates Redox balance. a. $3 c$ treated HT-29 cells were incubated with c-H2-DCFDA for 30 min. The fluorescence of the oxidized DCF was measured using Fluorescence plate reader (left). HT-29 cells treated with $3 c$ were incubated with c- $\mathrm{H}_{2}$ DCFDA for 15 min. Fluorescence of oxidized DCF was measured by flow cytometry (right) b. HT-29 cells were treated with $3 c$ for different time points and then incubated with $\mathrm{c}-\mathrm{H}_{2}$ DCFDA for 15 min. Fluorescence of oxidized DCF was determined using flow cytometry. $\mathbf{c}$. NAC pre-treated cells were incubated with $3 \mathrm{c}$ for $24 \mathrm{~h}$ followed by incubation with c-H2-DCFDA for $30 \mathrm{~min}$. Fluorescence was measured and results expressed as mean \pm SD of 3 determination. $\mathbf{d}$. HT-29 cells were treated with $3 \mathrm{c}$ for different time points and incubated with rhodamine 123 and analyzed by flow cytometry. e. Cells were analyzed for GSH levels using DTNB and presented as nmol/mg protein (left). $3 c$ treated cells were analyzed for NADPH levels (right). The results are expressed as the NADPH levels (mean \pm SD of 3 determinations)

PARP can be cleaved by many caspases and is the main cleavage target of caspase-3. Our results further demonstrate that $3 \mathrm{c}$ induces the cleavage of PARP as shown by an increase in cleaved PARP in HT-29 cells (Fig. 4a) and in SW620 cells (Fig. 4b). These results suggest that compound $3 c$ induces apoptosis by release of cytochrome c, caspase activation and cleavage of PARP.

To determine whether the 3c-induced apoptotic markers observed in human colon cancer cells is mediated by elevated ROS levels, we examined the activation of cleaved PARP and upregulation of cytochrome $\mathrm{c}$ by $3 \mathrm{c}$ in cells pretreated with NAC. HT-29 cells were pretreated with or without $5 \mathrm{mM} \mathrm{NAC}$ for $1 \mathrm{~h}$ and were then subjected to $3 \mathrm{c}$ treatment for an additional $24 \mathrm{~h}$. As expected, NAC abolished the activation of PARP by 3c (Fig. 4e). Similarly NAC was found to inhibit the release of cytochrome $\mathrm{c}$ into the cytosol (Fig. 4e). Similar results were obtained with SW620 cells (Fig. 4f).

\section{$3 \mathrm{c}$ inhibited cell migration}

The potential of cancer cells to migrate is of importance for cancer metastasis. To assess the effect of $3 \mathrm{c}$ on cell migration, we employed the SW620 colorectal cancer cells which are derived from late stage metastatic cancer. We analyzed the ability of these cells to migrate through porous membrane using xCELLigence system for real time data recording of cell migration. In the cell migration assay of the xCELLigence system, cells with higher potential to migrate attach to the bottom side of the membrane in the top chamber. This attachment of migrated cells increases the 


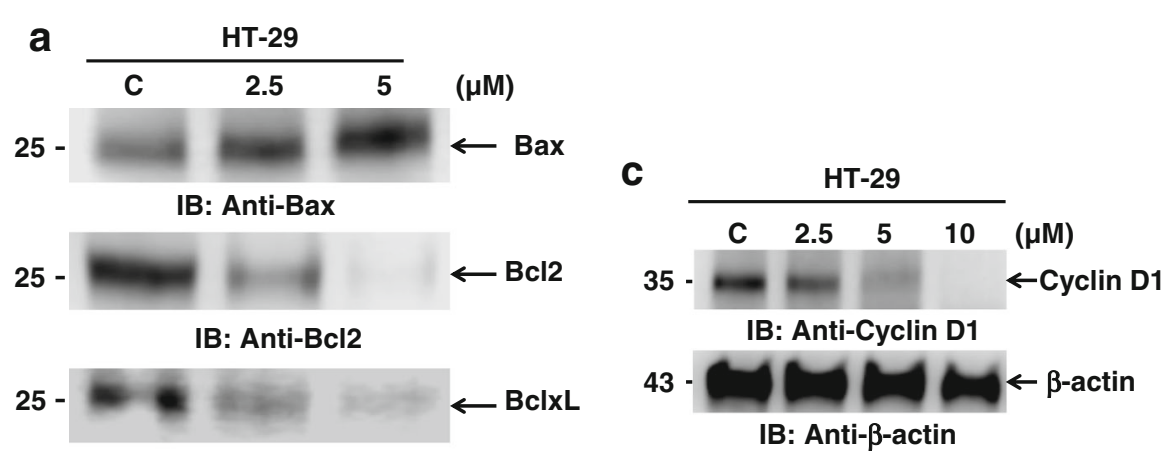

IB: Anti-BclxL

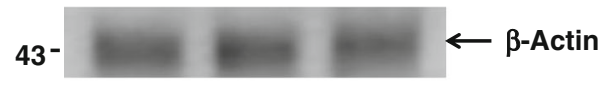

IB: Anti- $\beta$-Actin

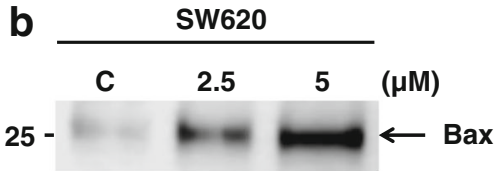

IB: Anti-Bax

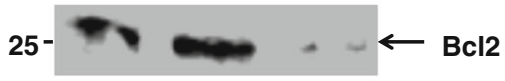

IB: Anti-Bcl2

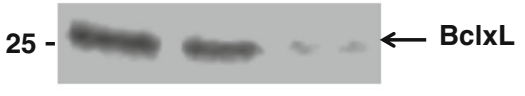

IB: Anti-BclxL

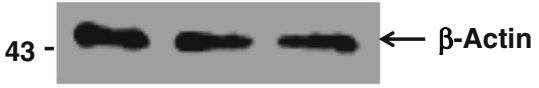

IB: Anti- $\beta$-Actin

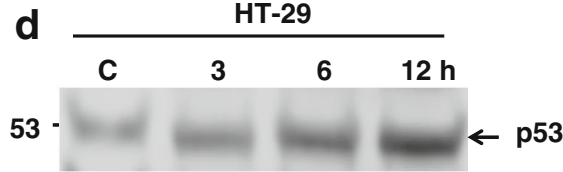

IB: Anti-p53

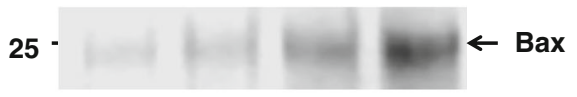

IB: Anti-Bax

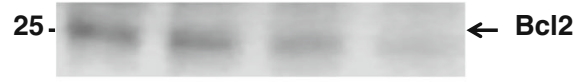

IB: Anti-Bcl2

43.

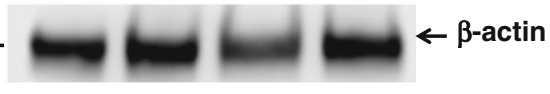

IB: Anti- $\beta$-Actin

Fig. 33 c alters Bcl2 family proteins and inhibits Cyclin D1. a. HT-29 cells were treated with different concentration of $3 c$ for 24 h. Cell lysates were immunoblotted with the indicated antibodies. b. SW620 cells were treated with various concentration of $3 c$ for 24 h. Cell lysates were immunoblotted with indicated antibodies. c. Total cell lysate from HT-29 cells treated with different concentration of 3c, were immunoblotted with the indicated antibodies. $\mathbf{d}$. Cells were treated with $3 \mathrm{c}$ for different time points, total cell lysates were immunoblotted with the indicated antibodies

electrical impedance. 3c induced a delay of signals as compared to control (Fig. 5a). This decrease in cell migration was also dose dependent. Integrating the area under the signal curves of three independent experiments showed a significant reduction in cell migration by $3 \mathrm{c}$ as compared to untreated controls. These results confirmed that $3 \mathrm{c}$ induces apoptosis and also has anti-migration effects. To further explore the effect of $3 \mathrm{c}$ on cell migration using a different approach, the wound healing assay was used to study HT29 colorectal cancer cells. Cell migration was evaluated by monitoring the closing of an applied scratch on a cell monolayer. As shown, wound healing was significantly impaired in 3c treated HT-29 cells as compared to control cells (Fig. 5b), suggesting that $3 \mathrm{c}$ inhibits cell motility and thereby cell migration in colorectal cancer cells. To further elucidate the mechanism by which $3 \mathrm{c}$ inhibits the cell migration, the epithelial to mesenchymal transition (EMT) is an essential step towards tumor invasion, migration and metastasis [25]. During EMT, expression of E-cadherin and vimentin is decreased and increased respectively. The TGF $\beta$-Smad pathway plays an important role in cell proliferation, differentiation, adhesion, EMT, migration and angiogenesis [25]. TGF $\beta$ treatment decreased E-cadherin expression in HT-29 cells. 3c treatment of cells reversed the TGF $\beta$-induced decrease in E-cadherin expression (Fig. 5c). 3c also inhibited Vimentin expression induced by TGF $\beta$ treatment. SW620 cells are partly mesenchymal and known to express both E-cadherin and Vimentin. 3c treatment alone inhibited E-cadherin expression without altering Vimentin expression though $3 c$ inhibits TGF $\beta$ induced Vimentin expression in SW620 cells (Fig. 5d). TGF $\beta$ phosphorylates Smad2 and Smad3 and forms a heterodimeric complex with Smad4 and translocate into the nucleus to regulate target genes. $3 \mathrm{c}$ inhibited TGF $\beta$ - 


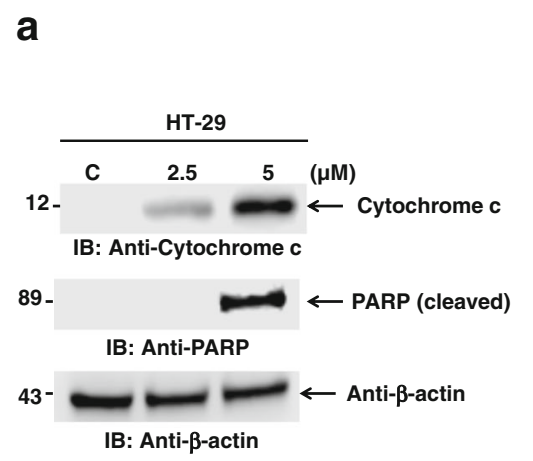

b

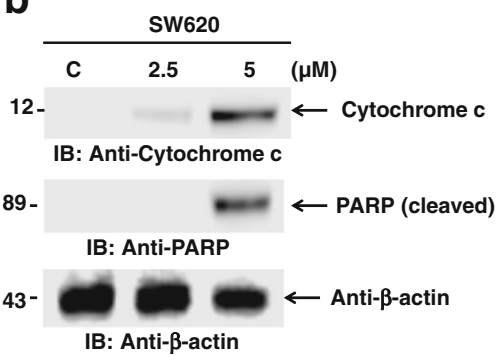

C

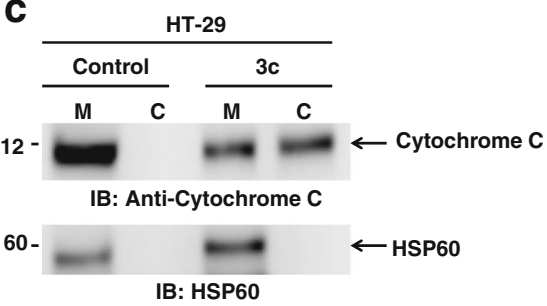

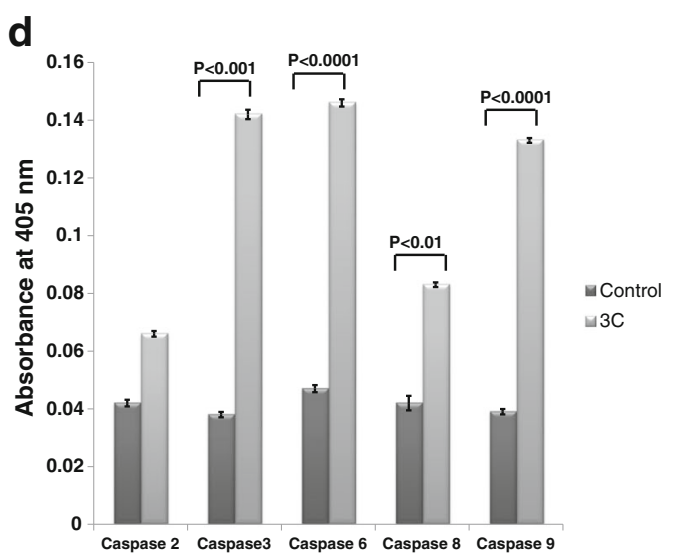

e

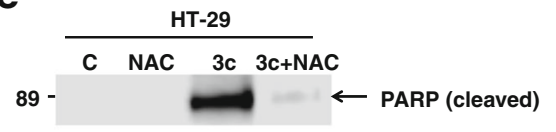

IB: Anti-PARP

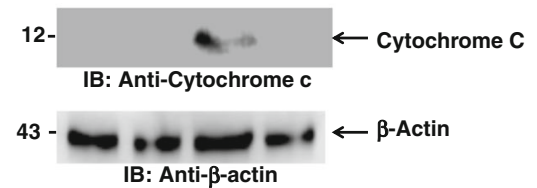

$\mathbf{f}$
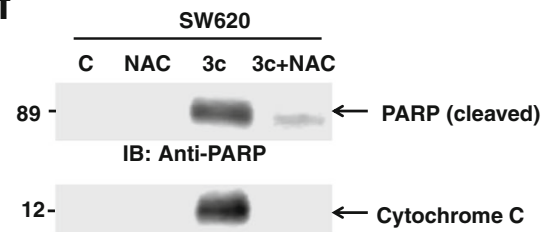

IB: Anti-Cytochrome c

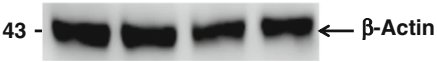

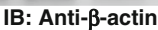

Fig. 4 3c activates cytochrome $c$ release, PARP and caspase cascade. a. HT-29 cytosolic extracts were immunoblotted with the indicated antibodies. b. SW620 cytosolic extracts were immunoblotted with the indicated antibodies. c. HT-29 cells were treated with 3c, mitochondrial and cytosolic extracts were isolated and immunoblotted with the indicated antibodies. $\mathbf{d}$. Cells were treated with $3 \mathrm{c}$ for $24 \mathrm{~h}$, cytosolic extracts were isolated and ELISA was performed for caspases activity. e. HT-29 cells were pre-treated with NAC (5 mM) for $1 \mathrm{~h}$ followed by $3 \mathrm{c}$ treatment. Cytosolic extracts were immunoblotted with the indicated antibodies. $\mathbf{f}$. SW620 cells were pre-treated with NAC (5 mM) for $1 \mathrm{~h}$ followed by $3 \mathrm{c}$ treatment. Cytosolic extracts were immunoblotted with the indicated antibodies

induced phosphorylation of Smad2 and Smad3 (Fig. 5e). Thus, these findings indicate that $3 \mathrm{c}$ inhibits TGF 3 induced Smad phosphorylation and EMT markers as mechanism for cell migration inhibition.

\section{Discussion}

We recently discovered a novel quinazolin-4-sulphomide derivative (3c) as a potential anti-tumor agent [19]. However the mechanism by which this compound acts remains unknown. In this study we shed new light on the mechanism by which 3c exerts its anti-cancer activity. Our results show that $3 \mathrm{c}$ induces apoptosis in human colorectal cancer cells by increased ROS production. ROS, which includes hydrogen peroxide, hydroxyl radical, and superoxides, are chemically active prooxidant molecules generated by incomplete reduction of oxygen [26]. Reactive oxygen species are involved in a variety of cellular physiology and pathology [27]. Anti-cancer agentsinduced ROS production that mediates the induction of apoptosis has been exploited in designing effective strategies for cancer therapeutics [26]. Many studies investigated the effects of both natural and synthetic anti-cancer agents which modulate ROS in colorectal cancer [28, 29]. $3 \mathrm{c}$ was found to increase ROS in colorectal cancer cells and was blocked by NAC. Our results support a model in which 3c-induced ROS production stimulates the intrinsic mitochondrial apoptotic pathway by decreasing outer mitochondrial membrane potential and thereby releasing 
a

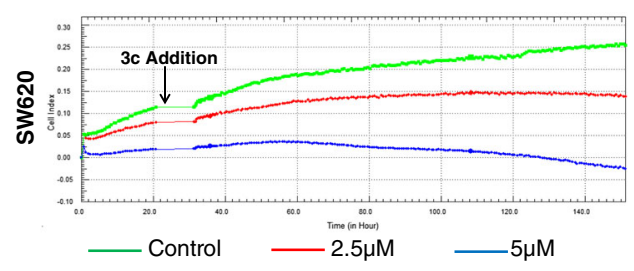

b

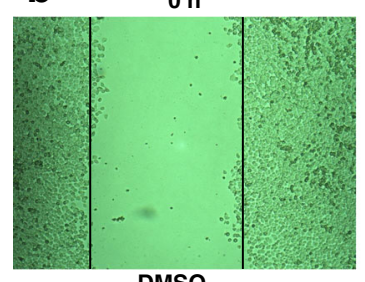

DMSO

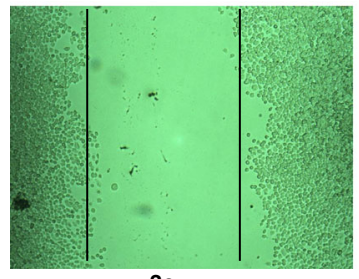

3c
$48 \mathrm{~h}$
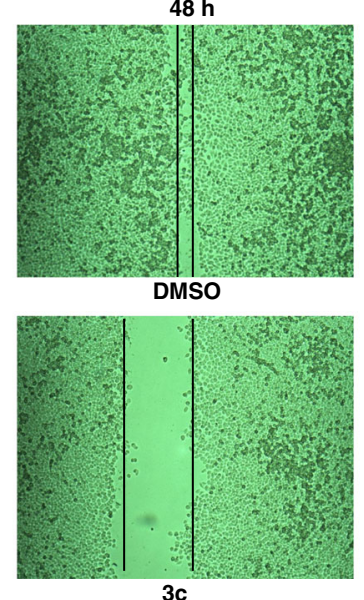

C

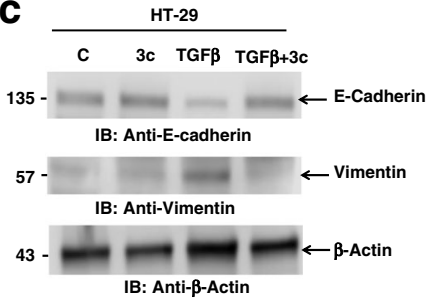

d

SW620

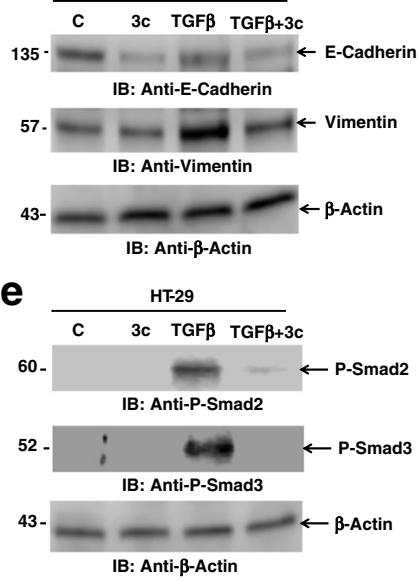

Fig. 5 Compound 3 inhibits migration of cancer cells. a. SW620 cells were seeded in the designated upper chamber and real time migrations of cells were monitored using XCELLigence RTCA-DP system. b. HT-29 cells were seeded into a 6-well plate and allowed to grow to $90 \%$ confluency in complete media. Cell monolayers were wounded by tips $(1 \mathrm{~mm})$ and washed with PBS. Media replaced with fresh media with either DMSO or $3 \mathrm{c}$ and put back into the incubator for $48 \mathrm{~h}$. Cells were monitored by microscope and digital images were captured. All the experiments were repeated three times and the representative images were shown. $\mathbf{c}$ : HT-29; d: SW620; e: HT-29 cells were pretreated with $3 c$ (5 $\mu \mathrm{M})$ and stimulated with TGF $\beta$, total cell lysates were immunoblotted with the indicated antibodies

cytochrome $\mathrm{c}$ that further activates caspase dependent signaling [30]. ROS are well known mediators of the intracellular signaling cascade known to play an important role in cancer drug discovery [31, 32]. They play critical role in the regulation of diverse functions, such as proliferation, apoptosis and transformation. When ROS levels reach a threshold point beyond the capacity of cellular antioxidant machinery it leads to oxidative stress, which in turn modulate mitochondrial membrane potential [33]. Several studies suggest that cancer cells are under increased oxidative stress associated with oncogenic transformation and increased ROS generation possibly due to its higher metabolic activity [34, 35]. A further increase in the ROS levels can make these malignant cells more vulnerable to cell death relative to untransformed cells. Thus, in cancer therapeutics, ROS signaling can be exploited to develop novel drugs to inhibit or kill cancer cells through its specific ROS signaling mechanism. Our newly developed compound $3 \mathrm{c}$ utilizes this mechanism in killing tumor cells. 3c treatment resulted in high level of ROS in HT-29 cells whereas NAC combined with $3 \mathrm{c}$ reduced ROS production and subsequently inhibited apoptosis. The molecular mechanism by which $3 \mathrm{c}$ induces elevated ROS production is presently not understood. Previous findings have reported that sulphonamide derivatives inhibit carbonic anhydrases activity [36-38]. 3c is known to inhibit the carbonic anhydrase IX and XII expression [19]. Some carbonic anhydrases have been reported to possess antioxidant property. Specifically, carbonic anhydrase III (CA III) and VII (CA VII) are known to have antioxidant characteristics [39-42]. This compound 3c may inhibit CA III and/ or CAVII expression and thereby increases ROS generation. Other mechanism may be involved as well like acting on electron transport chain or by abrogating key antioxidant systems in cells like depleting glutathione pool and/ or inhibiting superoxide dismutase (SOD) [43]. There are some anticancer agents for example elesclomol which is in clinical trial exerts its effect by inducing ROS but mechanism for ROS generation is not known [44]. Oxidative stress leads to decreased mitochondrial membrane potential. In this study $3 \mathrm{c}$ was also found to decrease mitochondrial membrane potential. We further showed that $3 \mathrm{c}$ induced PARP cleavage is blocked in the presence of NAC. Cytochrome $\mathrm{c}$ release induced by $3 \mathrm{c}$ was also inhibited by NAC, proving that indeed 3c induced apoptotic markers are ROS dependent. NADPH 
is required for the conversion of oxidized glutathione to reduced glutathione. $3 \mathrm{c}$ treatment resulted in decreased levels of NADPH and GSH reducing the cells antioxidation capacity and thus increasing oxidative stress.

We further uncovered the molecular pathway underlying 3c-induced apoptosis through upregulation of proapoptotic protein, Bax leading to the cytochrome c release from mitochondria. Cytochrome $\mathrm{c}$ release from mitochondria leads to the activation of caspase cascade which is essential in initiating apoptosis by anti-cancer agents [45]. 3c treatment resulted in an increase in caspase- 9 and effector caspase- 3 and 6 activities. Caspase- 9 is an initiator caspase in mitochondria mediated apoptosis pathway [46]. These findings indicate that $3 \mathrm{c}$ induces apoptosis through the intrinsic pathway. Certain caspases also target PARP for its cleavage into $24 \mathrm{kDa}$ and $89 \mathrm{kDa}$ fragments, rendering them incapable of DNA repair and leading to cell death. In this study, $3 \mathrm{c}$ was found to increase cleaved PARP levels. Cancer cells express series of anti-apoptotic proteins such as Bcl2 and BclxL. Overexpression of these anti-apoptotic proteins inhibits apoptosis and promotes cancer cell survival [47]. The anti-apoptotic Bcl2 protein has been demonstrated to be overexpressed in colorectal cancers [48]. According to Bonnotte et al., Bcl2 mediated apoptosis inhibition restores the tumorigenicity of colon tumors [49]. The reduced expression of $\mathrm{Bcl} 2$ and $\mathrm{BclxL}$ in our investigation suggests that 3c-induced apoptosis is mediated by an inhibition of these proteins. Zhu and colleagues have shown that induction of Bax expression is essential for deathreceptor mediated apoptosis in colon cancer cells [50]. Tumor suppressor p53 is a known regulator of $\mathrm{Bcl} 2$ and Bax gene expression [24]. p53 may be involved in the 3cinduced Bax expression and downregulation of $\mathrm{Bcl} 2$ expression. 3c was found to increase p53 and Bax expression in a time dependent manner similarly decreasing Bcl2 expression. This finding may provide a mechanism for 3cinduced alterations of $\mathrm{Bcl}$ family protein by involving $\mathrm{p} 53$ transcription factor. Our observation of elevated Bax expression and an increase in caspase- 8 activity in 3c treated colorectal cancer cells may suggest involvement of extrinsic apoptotic pathway as well. However, it is possible that caspase-8 through cleavage of BH3-only protein, BID also activates intrinsic apoptosis pathway [51]. Additionally we observed an important property of $3 c$ to inhibit cancer cell migration that would have a significant clinical benefit in controlling invasion and metastasis. Epithelial to mesenchymal transition (EMT) is essential process for cell invasion and migration [25]. 3c was found to alter the expression of EMT markers like E-Cadherin and Vimentin. TGF $\beta$-smad pathway is known to induce EMT in cancer cells. This compound of interest, 3c inhibits TGF $\beta$-induced smad pathway in colorectal cancer cells. These findings thus indicate that 3c-induced inhibition of cell migration is mediated by TGF $\beta$-smad pathway.

\section{Conclusions}

The present results indicate that compound 3c 1) induces apoptosis by increasing ROS levels, 2) increases p53 expression modulate Bcl 2 family proteins expression, 3) leads to PARP cleavage and cytochrome c release 4) reduces NADPH and GSH levels and 5) suppresses cancer cell migration by altering EMT markers and inhibits TGF $\beta$ dependent phosphorylation of Smads. These in-vitro results suggest that $3 \mathrm{c}$ is an attractive candidate for further investigation as potential anticancer agent. More studies are needed for the combination studies with other known therapeutics and for the in-vivo effect of 3c. A deeper understanding of the molecular mechanism of anti-cancer activity of $3 \mathrm{c}$ will allow for precise treatment regimens and combination therapeutics.

\section{Additional file}

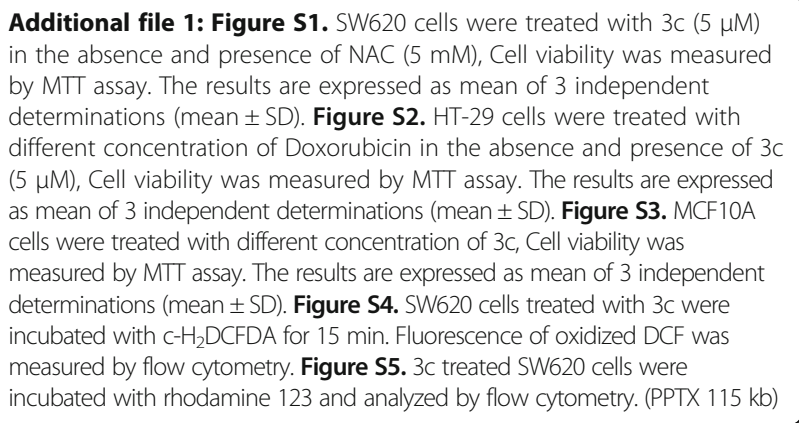

\section{Abbreviations}

CRC: Colorectal cancer; DCFDA: 2',7'-dichlorodihydrofluorescein diacetate; DMSO: Dimethyl sulfoxide; DTNB: 5,5-dithio-bis-(2-nitrobenzoic acid); EDTA: Ethylenediaminetetraacetic acid; GSH: Glutathione; MTT: 3-(4, 5dimethylthiazolyl-2)-2, 5diphenyltetrazolium bromide; NAC: N-acetylcysteine; NADPH: Nicotinamide adenine dinucleotide phosphate; PI: Propidium iodide; ROS: Reactive oxygen species.

\section{Acknowledgments}

We would like to thank Yahya Al-Qahtani for providing help with western blotting. The authors would like to thank for the support provided and continuous encouragement by the Vice Deanship Research Chair, Deanship of Scientific Research, King Saud University, Riyadh, Saudi Arabia.

Funding

The authors thank the Deanship of Scientific Research, King Saud University, Riyadh, KSA for the financial support.

\section{Availability of data and material \\ The entire data for this research is included in this article and its supplementary information.}

\section{Authors' contributions}

Conception and design of experiments: RA KA MAVM AA, Acquisition of data: MAVM AM AZ, Analysis and interpretation of data: OO ZK MA RA, Drafting of manuscript: RA MA ZK OO, Critical reading and revision: KA MAVM AZ AA MA RA, All authors approved the final manuscript.

\section{Competing interests}

The authors declare that they have no competing interest in this work.

Consent for publication

Not Applicable. 


\section{Ethics approval and consent to participate}

Not Applicable.

\begin{abstract}
Author details
${ }^{1}$ Colorectal Research Center, Department of Surgery, King Khalid University Hospital College of Medicine, King Saud University, PO BOX 7805 (37), Riyadh, Saudi Arabia. ${ }^{2}$ Department of Chemistry, Kulliyyah of Science, International Islamic University, P.O. Box 14125710 Kuantan, Malaysia. ${ }^{3}$ Stem Cell Unit, Department of Anatomy, King Khalid University Hospital College of Medicine, King Saud University, Riyadh, Saudi Arabia. ${ }^{4}$ Genome Research Chair, Department of Biochemistry, College of Science, King Saud University, Riyadh, Saudi Arabia.
\end{abstract}

Received: 9 August 2016 Accepted: 15 December 2016

Published online: 03 January 2017

\section{References}

1. Colorectal Cancer Facts \& Figures 2014-2016. American Cancer Society 128; http://www.cancer.org/acs/groups/content/documents/document/ acspc-042280.pdf

2. O'Connell JB, Maggard MA, Ko CY. Colon cancer survival rates with the new American Joint Committee on Cancer sixth edition staging. J Natl Cancer Institute. 2014;96:1420-5.

3. Lao W, Grady WM. Epigenetics and colorectal cancer. Nat Rev Gastroenterol Hepatol. 2011;8:686-700.

4. Ponnurangam S, Standing D, Rangarajan P, Subramaniam D. Tandutinib inhibits the Akt/mTOR signaling pathway to inhibit colon cancer growth. Mol Cancer Ther. 2013;12:598-609.

5. Zhang X, Wang X, Wu T, Li B, Liu T, Wang R, Liu Q, et al. Isoliensinie induces apoptosis in triple negative human breast cancer cells through ROS generation and p38 MAPKJJNK activation. Sci Rep. 2015;5:12579.

6. Circu ML, Aw TY. Reactive oxygen species, cellular redox systems, and apoptosis. Free Radic Biol Med. 2010;48:749-62.

7. Trachootham D, Alexandre J, Huang P. Targeting cancer cells by ROSmediated mechanisms: a radical therapeutic approach? Nat Rev Drug Discov. 2009;8:579-91.

8. Larocque K, et al. Novel analogue of colchicine induces selective pro-death autophagy and necrosis in human cancer cells. PLoS One. 2014;9:e87064.

9. Kim $\mathrm{AD}$, et al. A ginseng metabolite, compound $\mathrm{K}$, induces autophagy and apoptosis via generation of reactive oxygen species and activation of JNK in human colon cancer cells. Cell Death Dis. 2013;4:e750.

10. Kim JY, et al. Panaxydol induces apoptosis through an increased intracellular calcium level, activation of JNK and p38 MAPK and NADPH oxidase-dependent generation of reactive oxygen species. Apoptosis. 2011;16:347-58.

11. Ramiro-Cortes Y, Guemez-Gamboa A, Moran J. Reactive oxygen species participate in the p38-mediated apoptosis induced by potassium deprivation and staurosporine in cerebellar granule neurons. Int J Biochem Cell Biol. 2011:43:1373-82.

12. Hsieh CJ, et al. Arctigenin, a dietary phytoestrogen, induces apoptosis of estrogen receptor-negative breast cancer cells through the ROS/p38 MAPK pathway and epigenetic regulation. Free Radic Biol Med. 2014;67:159-70.

13. Chetram MA, Bethea DA, Odero-Marah VA, Don-Salu-Hewage AS, Jones KJ Hinton CV. ROS-mediated activation of AKT induces apoptosis via pVHL in prostate cancer cells. Mol Cell Biochem. 2013;376:63-71.

14. Hussain AR, Uddin S, Bu R, Khan OS, Ahmed SO, Ahmed M, et al. Resveratrol suppresses constitutive activation of AKT via generation of ROS and induces apoptosis in diffuse large B cell lymphoma cell lines. PLoS One. 2011;6:e24703.

15. Petros JA, Baumann AK, Ruiz-Pesini $E$, et al. mtDNA mutations increase tumorigenicity in prostate cancer. Proc Natl Acad Sci U S A. 2005;102:719-24.

16. Huang $P$, Feng $L$, OldhamEA KMJ, Plunkett W. Superoxide dismutase as a target for the selective killing of cancer cells. Nature. 2000;407:390-5.

17. Pelicano $H$, Feng $L$, Zhou $Y$, et al. Inhibition of mitochondrial respiration: a novel strategy to enhance drug-induced apoptosis in human leukemia cells by a reactive oxygen species-mediated mechanism. J Biol Chem. 2003;278: 37832-9.

18. TrachoothamD ZY, Zhang $\mathrm{H}$, et al. Selective killing of oncogenically transformed cells through a ROS-mediated mechanism by h-phenylethyl isothiocyanate. Cancer Cell. 2006;10:241-52.

19. Alafeefy AM, Ahmad R, Abdulla M, Eldehna WM, Al-Tamimi AM, Abdel-Aziz HA, Al-Obaid O, et al. Development of certain new 2-substituted-quinazolin- 4-yl-aminobenzenesulfonamide as potential antitumor agents. Eur J Med Chem. 2016:109:247-53.

20. Ahmad R, Raina D, Trivedi V, Ren J, Rajabi H, Kharbanda S, Kufe D. MUC1 oncoprotein activates the IkappaB kinase beta complex and constitutive NFkappaB signaling. Nat Cell Biol. 2007;9:1419-27.

21. Yin J-J, Sharma S, Shumyak SP, Wang Z-W, Zhou Z-W, et al. Synthesis and biological evaluation of novel folic acid receptor-Targeted, b-CyclodextrinBased drug complexes for cancer treatment. PLOS One. 2013;8:e62289.

22. Yin L, Kosugi M, Kufe D. Inhibition of the MUC1-C oncoprotein induces multiple myeloma cell death by down regulating TIGAR expression and depleting NADPH. Blood. 2012;119:810-6.

23. Xiao-Lan L, Zhou J, Zit-Liang C, Jing-Yuan C, Zhi-Rong C, Wee-Joo C. Prima1 met (APR-246) inhibits growth of colorectal cancer cell with different p53 status through distinct mechanisms. Oncotarget. 2015;6:36689-99.

24. Miyashita T, Krajewski S, Krajewska M, Wang HG, Lin HK, Liebermann DA, Hoffmann B, Reed JC. Tumor Suppresssor p53 is a regulator of bcl2 and bax gene expression in vitro and in vivo. Oncogene. 1994;9:1799-805.

25. Wang Z, Li Y, Sarkar FH. Siganling mechanism(s) of reactive oxygen species in Epithelial to Mesenchymal Transition Reminiscent of cancer stem cells in tumor progression. Curr Stem Cell Res Ther. 2010;5:74-80.

26. Sreevalsan S, Safe S. Reactive oxygen species and colorectal cancer. Curr Colorectal Cancer Rep. 2013;9:350-7.

27. Dickinson $\mathrm{BC}$, Chang $\mathrm{CJ}$. Chemistry and biology of reactive oxygen species in signaling or stress responses. Nat Chem Biol. 2011;7(8):504-11.

28. Yogosawa S, Yamada Y, Yasuda S, Sun Q, Takizawa K, Sakai T. Dehydrozingerone, a structural analogue of curcumin, induces cell-cycle arrest at the G2/M phase and accumulates intracellular ROS in HT-29 human colon cancer cells. J Nat Prod. 2012;75:2088-93.

29. Maillet A, Yadav S, Loo YL, Sachaphibulkij K, Pervaiz S. A novel Osmiumbased compound targets the mitochondria and triggers ROS-dependent apoptosis in colon carcinoma. Cell Death Dis. 2013;6(4):185.

30. Ryter SW, Kim HP, Hoetzel A, Park JW, Nakahira K, Wang X, Choi AM. Mechanisms of cell death in oxidative stress. Antioxid Redox Signal. 2007:9:49-89.

31. Martindale JL, Holbrook NJ. Cellular response to oxidative stress: signaling for suicide and survival. J Cell Physiol. 2002;192:1-5.

32. Hussain SP, Hofseth $L$, Harris CC. Radical causes of cancer. Nat Rev Cancer. 2003;3:276-85.

33. Ly JD, Grubb DR, Lawen A. The mitochondrial membrane potential (Dcm) in apoptosis; an update. Apoptosis. 2003;8:115-28.

34. Behrend L, Henderson G, Zwacka RM. Reactive oxygen species in oncogenic transformation. Biochem Soc Trans. 2003;31:1441-4.

35. Kang D, Hamasaki N. Mitochondrial oxidative stress and mitochondrial DNA. Clin Chem Lab Med. 2003:41:1281-8.

36. McDonald C, Winum J-Y, Supuran CT, Dedhar S. Recent developments in targeting carbonic anhydrase IX for cancer therapeutics. Oncotargets. 2012; 3(1):84-97.

37. Said HH, Hagemann C, Carta F, Katzer A, et al. Hypoxia induced CA9 inhibitory targeting by two different sulphonamide derivatives including Acetazolamide in human glioblastoma. Bioorg Med Chem. 2013:21(13): 3949-57.

38. Gieling RG, Parker CA, De Costa LA. Inhibition of carbonic anhydrase activity modifies the toxicity of doxorubicin and melphalan in tumor cells in vitro. J Enzym Inhib Med Chem. 2013;28:360-9.

39. Räisänen $S R$, Lehenkari $P$, Tasanen $M$, Rahkila $P$, Härkönen $P L$, Väänänen HK. Carbonic anhydrase III protects cells from hydrogen peroxide-induced apoptosis. FASEB J. 1999;13(3):513-22.

40. Zimmerman UJ, Wang P, Zhang X, Bogdanovich S, Forster R. Anti-oxidative response of carbonic anhydrase III in skeletal muscle. IUBMB Life. 2004;56(6):343-7.

41. Truppo E, Supuran CT, Sandomenico A, Vullo D, Innocenti A, Di Fiore A, Alterio V, De Simone G, Monti SM. Carbonic anhydrase VII is Sglutathionylated without loss of catalytic activity and affinity for sulfonamide inhibitors. Bioorg Med Chem Lett. 2012;22(4):1560-4.

42. Del Giudice R, Monti DM, Truppo E, Arciello A, Supuran CT, De Simone G, Monti SM. Human carbonic anhydrase VII protects cells from oxidative damage. Biol Chem. 2013;394(10):1343-8.

43. Kasiappan R and Safe S. ROS-Inducing agents for cancer chemotherapy. ROS. 2016;1 (1):22-37. http://dx.doi.org/10.20455/ros.2016.805.

44. Kirshner JR, He S, Balasubramanyam V, Kepros J, Yang CY, Zhang M, Du Z, Barsoum J, Bertin J. Elesclomol induces cancer cell apoptosis through oxidative stress. Mol Cancer Ther. 2008:7(8):2319-27.

45. Lowe SW, Lin AW. Apoptosis in cancer. Carcinogenesis. 2000;21:485-95. 
46. Burz C, Berindan-Neagoe I, Balacescu O, Irimie A. Apoptosis in cancer:key molecular signaling pathways and therapy targets. Acta Oncol. 2009;48:811-21.

47. Cherbonnel-Lasserre C, Dosanjh MK. Suppression of apoptosis by overexpression of $\mathrm{BCl} 2$ or $\mathrm{BClXL}$ promotes survival and mutagenesis after oxidative damage. Biochimie. 1997;79:613-7.

48. Zhu S, Li T, Tan J, et al. Bax is essential for death receptor-mediated apoptosis in human colon cancer cells. Cancer Biother Radiopharm. 2012;27:577-81.

49. Valassiadou KE, Stefanaki K, Tzardi M, et al. Immunohistochemical expression of p53, bcl2, mdm2 and waf1/p21 proteins in colorectal adenocarcinomas. Anticancer Res. 1997;17:2571-6.

50. Bonnotte B, Favre N, Moutet M, et al. Bcl2 mediated inhibition of apoptosis prevents immunogenicity and restores tumorigenicity of spontaneously regressive tumors. J Immunology. 1998;161:1433-8.

51. Mcllwain DR, Berger T, Mak TW. Caspase functions in cell death and disease. Cold Spring Harb Perspect Biol. 2013;5(4):a008656.

Submit your next manuscript to BioMed Central and we will help you at every step:

- We accept pre-submission inquiries

- Our selector tool helps you to find the most relevant journal

- We provide round the clock customer support

- Convenient online submission

- Thorough peer review

- Inclusion in PubMed and all major indexing services

- Maximum visibility for your research

Submit your manuscript at www.biomedcentral.com/submit
Biomed Central 